\title{
Effect of chaotropic anions on the sodium transport by the Na,K-ATPase
}

Received: 12 July 2005/ Revised: 14 October 2005/ Accepted: 18 October 2005 / Published online: 16 November 2005

\begin{abstract}
The effect of choline iodide, bromide and chloride on the kinetics of the electrogenic sodium transport by the Na,K-ATPase was investigated in a model system of ATPase-containing membrane fragments adsorbed on the lipid bilayer membrane. The kinetic parameters of $\mathrm{Na}^{+}$transport were determined from short circuit currents after fast release of ATP from its caged precursor. The falling phase of the current transients could be fitted by a single exponential with the time constant, $\tau_{2}$. Its temperature dependence allowed an estimation of the activation energy of the rate-limiting reaction step, the conformation transition $E_{1} / E_{2}$. Choline iodide and bromide caused a decrease of the activation energy as well as the overall rate of the process expressed as the pre-exponential factor $A$ of the Arrhenius equation. If choline iodide or bromide were present on the cytoplasmic and extracellular sides of the protein, the temperature dependent changes were more pronounced than when present on the cytoplasmic side only. These results can be explained by an effect of the anions on water structure on the extracellular surface of the protein, where a deep access channel connects the ion-binding sites with the solution. Chloride ions also caused a deceleration of the electrogenic transport, however, in contrast to iodide or bromide, they did not affect the activation energy, and were more effective when added on the cytoplasmic side. This effect can be explained by asymmetric screening of the negative surface charges which leads to a transmembrane electric potential that modifies the ion transfer.
\end{abstract}

A. G. Ayuyan · V. S. Sokolov · A. A. Lenz

A.N. Frumkin Institute of Physical Chemistry

and Electrochemistry, Russian Academy of Sciences,

31 Leninski Prospect, 119071 Moscow, Russia

H.-J. Apell ( $\square)$

Department of Biology, University of Konstanz,

Fach M635, 78457 Konstanz, Germany

E-mail: h-j.apell@uni-konstanz.de
Keywords Ion pump - Charge movement . Kinetics $\cdot$ Caged ATP

\section{Introduction}

The Na,K-ATPase is one of the most important and widespread proteins in the animal kingdom, which performs active transport of $\mathrm{Na}$ and $\mathrm{K}$ ions across the cytoplasmic membrane of the cells. Intensive studies were conducted during recent years and resulted in a significant progress in elucidating pump function. Conformational rearrangements of the Na,K-ATPase play a key role in the transport process. While the pump runs through its cycle, alternating access to the ion binding sites through channel-like structures on both sides of the membrane domain enables in the $\mathrm{E}_{1}$ conformation an exchange of three $\mathrm{Na}^{+}$against two $\mathrm{K}^{+}$ions on the cytoplasmic side and vice versa on the extracellular side in the P-E $\mathrm{E}_{2}$ conformation (Apell 2003; Pavlov and Sokolov 2000).

It has been known for more than 15 years that flat membrane fragments with a high density of $\mathrm{Na}, \mathrm{K}-\mathrm{AT}$ Pase molecules (about 5,000 per $\mu \mathrm{m}^{2}$ ), isolated and purified from rabbit kidneys, can be adsorbed to bilayer lipid membranes (BLM). This system is convenient to investigate the ion-transport mechanism of the Na,KATPase (Borlinghaus et al. 1987; Fendler et al. 1985; Wuddel and Apell 1995). In such a setup, in the absence of potassium ions, short circuit current can be generated by the transfer of sodium ions through the membrane domain of the $\mathrm{Na}, \mathrm{K}$-ATPase towards the extracellular side of the membrane, which is facing the BLM. Such currents can be recorded as synchronized response to a fast ATP-concentration jump, which is produced by the release of ATP from its inactive precursor, caged ATP, upon an UV light flash. The triggered current transients have three characteristic phases: rise and decay of current in response to the flash, as well as third phase of a negative current component which is associated with the slow passive discharge of the electric capacity of the 
adsorbed fragments and of the BLM (Sokolov et al. 2001; Wuddel and Apell 1995). The transfer of $\mathrm{Na}^{+}$ions consists of several sequential steps, including binding of three $\mathrm{Na}^{+}$ions from the cytoplasmic side of the membrane, binding and hydrolysis of ATP, phosphorylation, $\mathrm{P}-\mathrm{E}_{1} / \mathrm{P}-\mathrm{E}_{2}$ conformation transition, and release of the ions on the extracellular side. Determination of the time constants of the three current phases does not provide sufficient information to obtain the rates of all the reaction steps, however, the rate of the slowest step controlling the time constant of the respective partial reaction can be identified. The conformation transition $\mathrm{P}-\mathrm{E}_{1} / \mathrm{P}-\mathrm{E}_{2}$ is supposed to be the slowest step of the ion transfer (Sokolov et al. 1998b). Although this interpretation is most widely adopted (Holmgren et al. 2000; Sokolov et al. 1998a; Wuddel and Apell 1995), there is an alternative point of view that the conformation transition controls the rising phase of the current signal (Fendler et al. 1993).

One method to solve this issue is to vary the rate of the conformational transition by the so-called "salt effect". It is well known that the conformation of macromolecules dissolved in water depends upon the ionic composition of the solution. The most likely explanation is that the energy of water molecules bound to the macromolecule-water interface depends on the presence of other substances in the solution. Some substances, known as chaotropes, increase the binding energy for water whereas other substances, referred to as cosmotropes, decrease it (Cacace et al. 1997). The effect of the chaotropic ions on the equilibrium between the two principal conformations of the Na,K-ATPase, $\mathrm{E}_{1}$ and P$E_{2}$, was demonstrated earlier by investigation of the effect of different salts on binding of inorganic phosphate to the Na-K-ATPase (Post and Suzuki 1991).

Chaotropic ions are known to slow down the electrogenic transport of sodium ions by the Na,K-ATPase in the model system consisting of a BLM with adsorbed $\mathrm{Na}, \mathrm{K}-\mathrm{ATPase}$ containing membrane fragments. Specifically, in the presence of different anions a deceleration of the kinetics of the short circuit current, and a shift of the frequency dependence of the admittance towards lower frequencies were observed (Ganea et al. 1999; Sokolov et al. 2001; Sokolov et al. 1998b). The magnitude of the effect depended on the anions present: the effectiveness of choline salts with different halogenoids increased in the sequence $\mathrm{Cl}^{-}<\mathrm{Br}^{-}<\mathrm{I}^{-}$corresponding to their position in the Hofmeister series (Sokolov et al. 2001). This finding can be explained by the effect of chaotropic anions on the rate of the conformation transition in the protein. Other explanations, however, could not be excluded so far, e.g. salt-induced changes of the aggregation state of the lipids surrounding the protein (Klodos et al. 1994), as well as non-specific screening of the charged groups of the protein, or competition of anions for the ATP binding site (Nørby and Esmann 1997).

Investigations of temperature dependencies of the salt effects can be used to prove that the effects are associated with the conformation transition of the $\mathrm{Na}, \mathrm{K}-\mathrm{ATPase}$. This rate is known to be highly sensitive to temperature, as indicated by the high-activation energy, which was estimated to be about $80 \mathrm{~kJ} / \mathrm{mol}$, based on the electric measurements with cardiomyocytes (Gadsby et al. 1992). If the choline salts affect the rate of the conformational transition, they may also affect the corresponding activation energy. Besides the membrane domain with 10 transmembrane helices, the $\mathrm{Na}, \mathrm{K}$ ATPase has a large cytoplasmic part that consists of three different domains that represent the enzymatic machinery, and a small extracellular part that is formed mostly by the $\beta$ subunit of the protein. Therefore, it may be expected that the chaotropic salts produce different effects on the two sides of the membrane. The Na,KATPase-containing membrane fragments are known to adsorb on the BLM with a preferred orientation, the extracellular membrane side facing the BLM. This property allows comparison of the effects of choline salts with various anions on the $\mathrm{Na}, \mathrm{K}-\mathrm{ATPase}$ on both sides of the membrane, when the salts are added to the solution either prior to the adsorption on BLM or after the tight adsorption has occurred. In the first case the salts can affect the ATPase on both sides of the membrane fragment, whereas in the second case only the cytoplasmic side is exposed to the bulk solution. (During the time period of the experiments the ions or ouabain do not penetrate into the narrow space of the contact area between membrane fragment and BLM (Borlinghaus et al. 1987; Fendler et al. 1985).) In the following presentation we will refer to a 'bilateral' or 'unilateral' addition of ions when the choline salts were added before or after adsorption of the membrane fragments, respectively.

In the present study, we have proven by measurements of the temperature dependency that the choline salts of chaotropic anions affect the activation energy of the slowest step of electrogenic transport, being much more effective when present on the extracellular side of the protein. This implies that the salts do affect the $\mathrm{P}-\mathrm{E}_{1} /$ $\mathrm{P}_{-} \mathrm{E}_{2}$ conformation transition rather than ATP hydrolysis, since hydrolysis occurs at the cytoplasmic side of the protein and has a different temperature dependence.

\section{Materials and methods}

Membrane fragments containing purified Na,K-ATPase were obtained from rabbit kidney according to Jorgensen procedure "C" (Jørgensen 1974). The membrane fragments are planar patches with the diameter of $0.2-1 \mu \mathrm{m}$, containing about $0.8 \mathrm{mg}$ of phospholipids and $0.2 \mathrm{mg}$ of cholesterol per $1 \mathrm{mg}$ of protein (Deguchi et al. 1977). The enzyme activity was $1,800-2,000 \mu \mathrm{mol}$ of inorganic phosphate released per $1 \mathrm{mg}$ of protein per hour at $37^{\circ} \mathrm{C}$. The aqueous suspension of the fragments with a protein concentration of about $3 \mathrm{mg} / \mathrm{ml}$ can be stored in a deep freezer for several months without notable loss of ATPase activity. For the experiments, the 
suspension was thawed and subsequently stored at $+4^{\circ} \mathrm{C}$ for less than 2 weeks.

BLMs were formed in a Teflon cuvette from a $15 \mathrm{mg} /$ $\mathrm{ml}$ solution of diphytanoyl lecithin (Avanti Polar Lipids, USA) in n-decane (Aldrich, USA). The electrolyte contained $\mathrm{NaCl}, \mathrm{MgCl}_{2}$ (Merck, Germany), imidazole (Serva, Germany), dithiothreitol (DTT, Sigma, USA), caged ATP (Calbiochem, USA). The basic buffer solution (which will be referred to as "standard") contained $150 \mathrm{mM} \mathrm{NaCl}, 30 \mathrm{mM}$ imidazole, $10 \mathrm{mM} \mathrm{MgCl}_{2}$, and $1 \mathrm{mM}$ EDTA at $\mathrm{pH} 6.5(\mathrm{HCl})$.

The electric current signals generated by the $\mathrm{Na}, \mathrm{K}$ ATPase were measured by the method described earlier (Borlinghaus et al. 1987). BLM were formed in a thermostated Teflon cuvette with two optically transparent windows for visual control of the membrane and for exposure to the UV light flash. After the BLM was formed in standard buffer, DTT was added to both compartments of the cuvette, then membrane fragments and caged ATP were added to the compartment opposite to the source of ultra-violet light (concentrations of the caged ATP and protein in the cuvette were $100 \mu \mathrm{M}$ and about $20 \mu \mathrm{g} / \mathrm{ml}$, respectively). The adsorption of the membrane fragments on the BLM was monitored by a decrease of the measured capacitance.

Current transients were recorded after the ATP release from caged ATP, triggered by the UV flash. A xenon flash lamp with sapphire window (FJ-249, EG\&G, USA) was used as UV-light source. The release of ATP caused a transient current generated by transfer of the $\mathrm{Na}^{+}$ions through the Na,K-ATPase from the (cytoplasmic) bulk solution to the space between BLM and membrane fragments (Borlinghaus et al. 1987). Multiple records were made with the same membrane under identical conditions and averaged in order to increase the signal/noise ratio. Typical time intervals between two light flashes were longer than $10 \mathrm{~min}$ to ensure recovery of the initial state of the Na,K-ATPase. Some experiments were made in the presence of apyrase, a soluble enzyme that hydrolyses the free ATP in the buffer and accelerates restoration of the initial state of the Na,K-ATPase (Borlinghaus et al. 1987). In these cases the interval between the flashes could be decreased to 3 min. The amplitude of the transient currents increased between the sequential flashes, as long as the adsorption of the fragments continued. The electrical signals generated by the Na,K-ATPase were recorded only after the current amplitude and the membrane capacitance became stable, which indicated that the adsorption process was completed (usually more than $2 \mathrm{~h}$ after addition of the membrane-fragment suspension).

The electric currents were measured with $\mathrm{Ag} / \mathrm{AgCl}$ electrodes, connected to the electrolyte in the membrane cuvette via salt bridges. The bridges were filled with the same solution as the cuvette. The resistance of the electrodes with the bridges did not exceed $30 \mathrm{k} \Omega$. In order to record the short circuit currents, one of the electrodes was grounded, and the other connected to the input of the operational amplifier Keithley 427 (USA). The output signal of the amplifier was recorded by a digital oscilloscope KDS-102 (Kawasaki, Japan), or L780 ADC/DAC board (Lcard, Russia), and transferred to a PC. The current trace was fitted by a sum of exponential functions with a computer program designed by the authors. The temperature in the cuvette was maintained by an electronic thermostat based on a Peltier element.

\section{Results}

A typical record of a short circuit current is shown in Fig. 1. The time dependence of the current was fitted by a sum of three exponentials to obtain the three time constants: $\tau_{1}, \tau_{2}$ and $\tau_{3}$. Only the time constants $\tau_{1}$ and $\tau_{2}$ were of interest for subsequent analysis, since the constant $\tau_{3}$ characterizes the slow, passive discharge of the membrane capacitances, and is not directly related to the function of the ion pump (Borlinghaus et al. 1987). The measurements were conducted at different temperatures in the range between 15 and $30^{\circ} \mathrm{C}$. The determined time constants at each temperature were plotted as Arrhenius plot, $\ln (1 / \tau)$ versus $1 / T$. Such a dependence of $\tau_{2}$ is shown in Fig. 2. The activation energy, $E_{a}$, was obtained from the slope of the linear fit of the data points in the Arrhenius plot. The pre-exponential, temperature-independent factor $A$ characterizes the vertical position of the data points in the Arrhenius equation:

$k=A \cdot \exp \left(-\frac{E_{\mathrm{a}}}{R} \cdot \frac{1}{T}\right)$,

where the rate, $k=1 / \tau$, is the reciprocal time constant of the corresponding exponential function. The activation energy of $\tau_{1}, E_{\mathrm{a}}$, which represents the rising phase of the current transient, was $98 \mathrm{~kJ} / \mathrm{mol}$ in the standard solution, whereas an $E_{\mathrm{a}}$ of $65 \mathrm{~kJ} / \mathrm{mol}$ was obtained for $\tau_{2}$ characterizing the falling phase. These values are in good agreement with previously reported results of measurements performed in the same model system under similar conditions (Fendler et al. 1993), where the activation energies were reported to be $101 \mathrm{~kJ} / \mathrm{mol}$ and $68 \mathrm{~kJ} / \mathrm{mol}$, respectively.

The amplitude of short circuit current decreased and its kinetics was slowed down in the presence of choline iodide (Fig. 1). This effect was reflected in an increase of the time constants, $\tau_{1}$ and $\tau_{2}$ as reported recently by the authors (Sokolov et al. 2001). It has been shown previously that this effect is caused by the anions and not by choline (Sokolov et al. 1998, 2001). In order to reveal the underlying mechanism, the effect of anions on the temperature dependence of the kinetical parameter was studied. Addition of iodide ions changed only insignificantly the process which controls the rising phase of the current transient with its time constant, $\tau_{1}$, (data not shown), whereas the effect on the falling phase, represented by $\tau_{2}$, was much more pronounced. Fig. $2 \mathrm{a}$ 


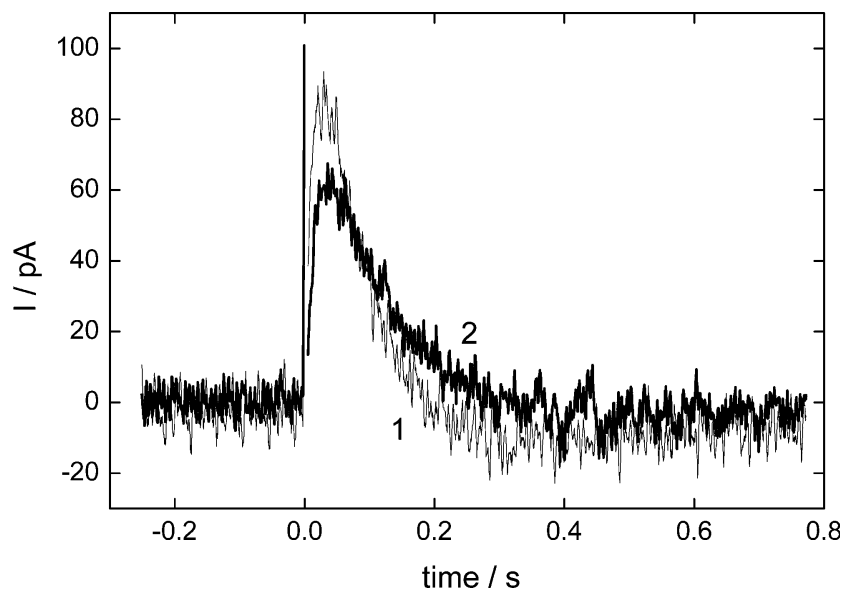

Fig. 1 Kinetics of the short circuit current caused by UV flash induced ATP-concentration jump before (1) and after (2) unilateral addition of $50 \mathrm{mM}$ choline iodide to the electrolyte. The curve was fitted by a sum of three exponentials that yields the following values of the time constants (ms): $\tau_{1}=17, \tau_{2}=34, \tau_{3}=309$ (1); $\tau_{1}=19$, $\tau_{2}=76, \tau_{3}=331$ (2). The BLM was formed from diphytanoyl phosphatidylcholine $(15 \mathrm{mg} / \mathrm{ml}$ in n-decane) in standard buffer at $24^{\circ} \mathrm{C}$ and had an area of about $0.3 \mathrm{~mm}^{2}$ (capacitance of $1,3 \mathrm{nF}$ ). After formation of the BLM, dithiothreitol was added as reducing agent to both compartments of the cuvette, and the suspension of the membrane fragments containing $\mathrm{Na}, \mathrm{K}$-ATPase (protein concentration about $30 \mu \mathrm{g} / \mathrm{ml})$ and caged ATP $(100 \mu \mathrm{M})$ were added to the compartment opposite of the light source

illustrates the temperature dependency of $1 / \tau_{2}$ at different choline-iodide concentrations $(0-60 \mathrm{mM})$ when the ions were added prior to the adsorption of the membrane fragments (bilateral addition). Choline iodide caused a decrease of the rate parameter $A$ (downwards shift of the data points) as well as a decrease of the activation energy $E_{\mathrm{a}}$. The parameters $A$ and $E_{\mathrm{a}}$ were found to change in a correlated manner as function of the choline iodide concentration. In Fig. $2 \mathrm{~b}$ each point corresponds to the values of $\ln (A)$ and $E_{\mathrm{a}}$, obtained from the fit of the temperature dependence of the $\mathrm{Na}^{+}$ transport by the Na,K-ATPase at the iodide concentration shown in Fig. 2a. As can be seen from Fig. 2b, $\ln (A)$ is linearly related to $E_{\mathrm{a}}$, which means that the temperature dependencies obtained at different choline iodide concentrations intersect in one point. The coordinates of this point, which can be easily deduced from the slope of the line on Fig. 2b, correspond to a temperature of $270 \mathrm{~K}$, which is close to freezing temperature of water.

If the bromide or iodide salts were added to the solution after complete adsorption of Na,K-ATPasecontaining fragments (unilateral addition), the changes of $A$ and $E_{a}$ were much smaller than in the case of bilateral addition (Fig. 3). Hence, these salts affect the protein more effectively when present on both the cytoplasmic and the extracellular sides. This observation indicates that the extracellular side is more sensitive to the effects of iodide.

One possible explanation of these salt effects is - as it was discussed in the literature - that the anions affect
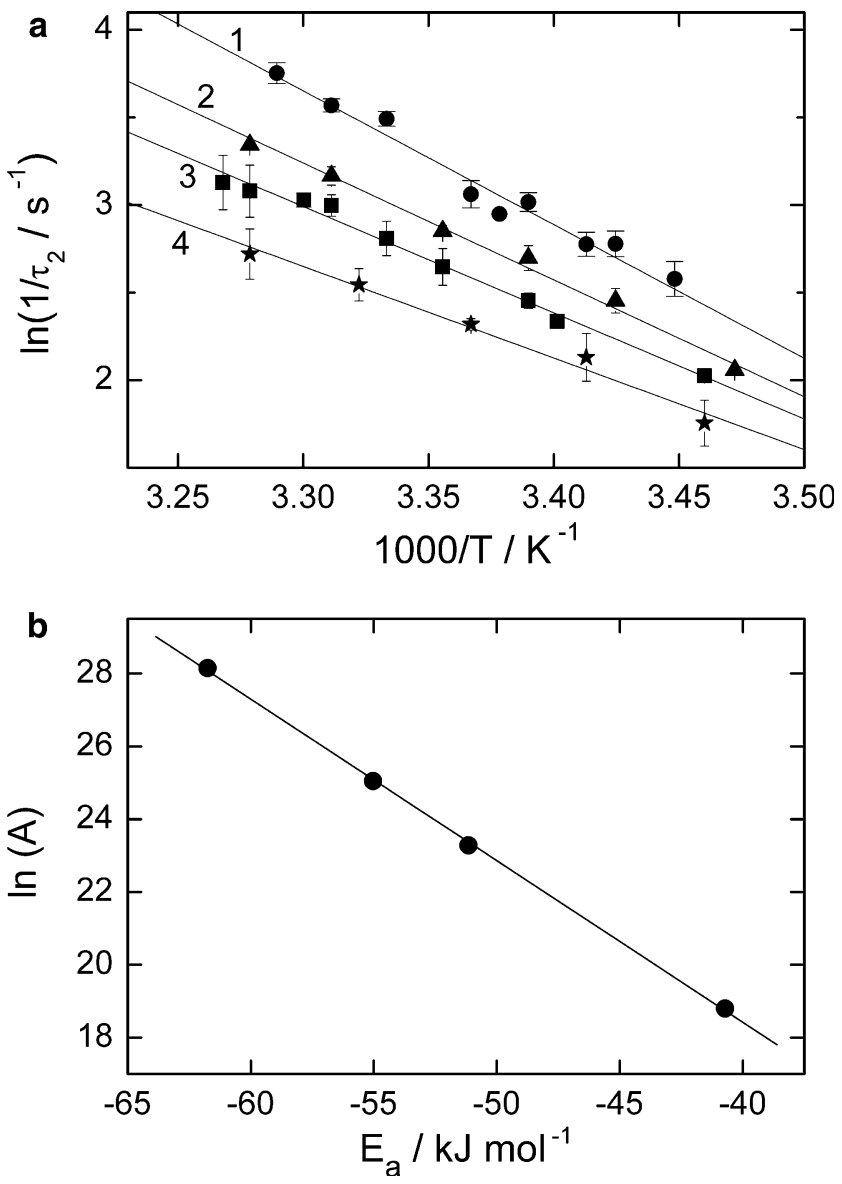

Fig. 2 a Temperature dependence of the reciprocal time constant of the exponential function approximating the decay phase of the short circuit current, $\left(1 / \tau_{2}\right)$, represented as Arrhenius plot. The measurements were performed in the standard buffer (trace 1), or after bilateral addition of several choline-iodide concentrations: $15 \mathrm{mM}$ (trace 2), $35 \mathrm{mM}$ (trace 3) and $60 \mathrm{mM}$ (trace 4). The lines fitted through the data correspond to the following values of the parameters of the Arrhenius equation, $E_{\mathrm{a}}(\mathrm{kJ} / \mathrm{mol})$ and $\ln (A)\left(\mathrm{s}^{-1}\right)$, respectively: 61.7 and 28.2 (0 iodide); 55.0 and $25.0(15 \mathrm{mM}) ; 51.1$ and $23.3(35 \mathrm{mM}) ; 40.7$ and $18.8(60 \mathrm{mM})$. b Correlation between $E_{\text {a }}$ and $\ln (A)$, as obtained from the fit of the data shown on panel (a). The linear correlation could be fitted by: $\ln (A)=0.67-0.44 \cdot E_{\mathrm{a}}$

ATP binding to the Na,K-ATPase or compete with ATP for the nucleotide-binding site (Ganea et al. 1999; Nørby and Esmann 1997). An alternative explanation would be that the anions screen the electrostatic field produced by the charged groups of the protein in the vicinity of the ATP-binding site (Fedosova et al. 2003; Nørby and Esmann 1997). In order to verify whether the effect of choline salts is caused by these reasons, we investigated the temperature dependency of $1 / \tau_{2}$ at variable intensities of the UV flash, thus varying the amount of ATP released from caged ATP. The results are presented in Fig. 4. A decrease of the flash intensity (or ATP concentration) affected the temperature dependence of $1 / \tau_{2}$ in a different manner when compared to addition of iodide. When the light intensity was reduced to $50 \%$, the slope of temperature dependence of $1 / \tau_{2}$ increased, and correspondingly, the activation energy grew from 


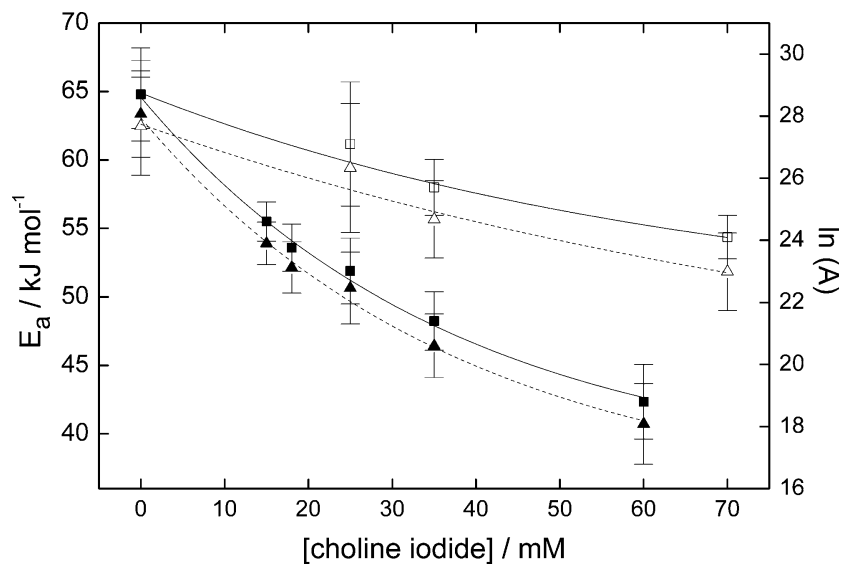

Fig. 3 Dependence of the activation energy, $E_{\mathrm{a}}$, (triangles) and preexponential factor (squares) on the concentration of choline iodide when added either bilateral, i.e. before addition of the membrane fragments (solid symbols), or unilateral, i.e. after completion of their adsorption on BLM (open symbols)

$62.3 \mathrm{~kJ} / \mathrm{mol}$ to $119.7 \mathrm{~kJ} / \mathrm{mol}$ in the temperature range between $21{ }^{\circ} \mathrm{C}$ and $31^{\circ} \mathrm{C}$. Addition of the iodide caused the slope to decrease (Fig. 2), and the activation energy to be reduce from $61.2 \mathrm{~kJ} / \mathrm{mol}\left(0 \mathrm{I}^{-}\right)$to $18.8 \mathrm{~kJ} / \mathrm{mol}$ $\left(60 \mathrm{mM} \mathrm{I}^{-}\right)$. Moreover, the intersection point of the fitting lines in the Arrhenius plot was about $28^{\circ} \mathrm{C}$ when the amount of ATP released was reduced by about a factor of two, while it was close to $0^{\circ} \mathrm{C}$ for all iodide concentrations between 0 and $60 \mathrm{mM}$.

When the temperature dependence of $1 / \tau_{2}$ was investigated in the presence of various choline-chloride concentration the slope of the Arrhenius plot (and accordingly the activation energy) was not significantly affected (not shown), and hence the (temperature independent) decrease of $1 / \tau_{2}$ at elevated chloride

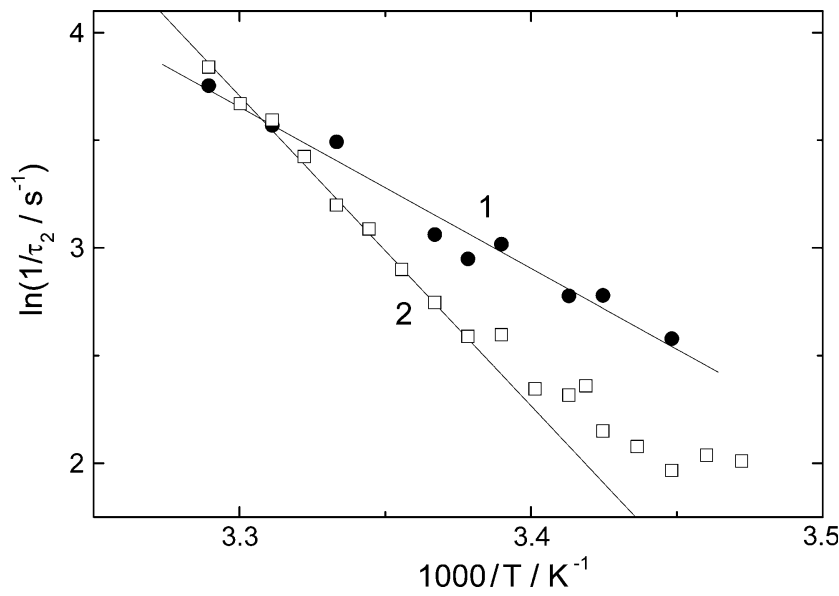

Fig. 4 Effect of the variation of the amount of ATP released on the temperature dependence of $1 / \tau_{2}$. Dataset 1 was obtained under standard conditions (as trace 1 in Fig. 2). Dataset 2 was obtained at a flash energy reduced by a factor of two, corresponding to a reduction of the amount of ATP released by about the same factor

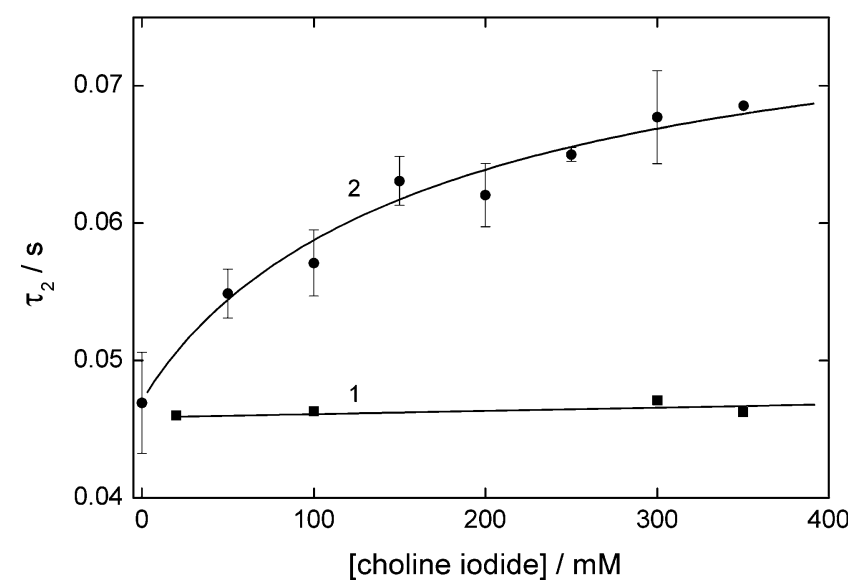

Fig. 5 Dependence of the time constant, $\tau_{2}$, of the fit to the decay of the short circuit current (cf. Fig. 1) on the concentration of choline chloride added to the cuvette either (1) before addition of the membrane fragments ("bilateral addition"), or (2) after completion of their adsorption on BLM ("unilateral addition"). $\mathrm{T}=24^{\circ} \mathrm{C}$

concentrations has to be attributed to a decrease of the pre-exponential factor $A$.

There was also a difference between the different anions in the experiments with bilateral and unilateral addition of the salts. Unilateral addition of choline chloride caused a more pronounced deceleration of the short circuit current kinetics than a bilateral addition. The value of $\tau_{2}$, increased only insignificantly, and $\tau_{1}$ remained constant in the case of a bilateral addition of choline chloride at concentrations up to $850 \mathrm{mM}$ (Sokolov et al. 2001). In the case of unilateral addition, $\tau_{2}$ increased significantly at concentrations above $50 \mathrm{mM}$ (Fig. 5). This observation is in contrast to the results obtained in the presence of iodide. When iodide

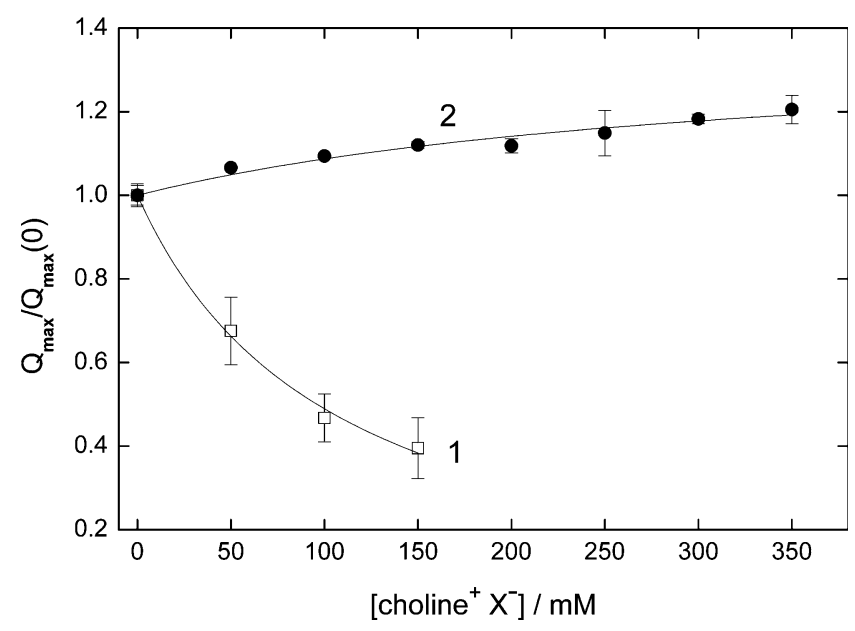

Fig. 6 Dependence of the charge transferred by the Na,K-ATPase on the concentration of choline iodide (1) or chloride (2) added to the cuvette after adsorption of the membrane fragments to the BLM ("unilateral addition"). The charge transfer was triggered by an ATP-concentration jump. The condition of the experiments is described in Fig. 1. The transported charge was defined as maximum of the integral of short circuit current, the values are normalized to the charge obtained before addition of choline salts 
was added unilaterally, $\tau_{2}$ developed as function of concentration similarly to the findings with chloride, i.e. $\tau_{2}=0.057 \pm 0.002 \mathrm{~s}$ at $100 \mathrm{mM}$, while $\tau_{2}$ increased to about $0.1 \mathrm{~s}$ at $100 \mathrm{mM}$ when iodide was added bilaterally (Sokolov et al. 2001). In contrast, chloride had no significant effect on $\tau_{2}=0.046 \pm 0.001 \mathrm{~s}$ when added bilaterally in concentrations between $50 \mathrm{mM}$ and $350 \mathrm{mM}$ (Fig. 5). This behavior, as it will be discussed below, can be explained rather by screening of surface charge than by a direct interaction of choline with the $\mathrm{Na}, \mathrm{K}-\mathrm{ATPase}$.

The experiments repeated with choline bromide instead of choline iodide showed that bromide gives the same effect as iodide (data not shown).

A difference between chloride on the one hand and iodide or bromide on the other hand was also found for the amount of charge moved across the membrane after an ATP-concentration jump. The amount of charge transported by the $\mathrm{Na}-\mathrm{K}-\mathrm{ATP}$ ase was determined as described before as a maximum value of the integrated short circuit current (Sokolov et al. 2001). When bromide or iodide were added, the amount of charge was significantly reduced to about $50 \%$ at $100 \mathrm{mM}$, whereas addition of even $350 \mathrm{mM}$ chloride led only to a slight increase of about 20\% (Fig. 6).

\section{Discussion}

We measured the temperature dependency of the characteristic time constants of the ATP-induced current transients, $\tau_{1}$ and $\tau_{2}$, in solutions containing choline salts of three different anions, chloride, bromide and iodide. The latter two anions affected the temperature dependence of $1 / \tau_{2}$ by significantly decreasing the activation energy of the rate-limiting reaction step. The effect of iodide was stronger than that of bromide. From the literature it is known that bromide and iodide anions are strong chaotropic agents, with iodide being notably more effective than bromide according to the Hofmeister series (Post and Suzuki 1991). Therefore, the observed effect of these ions may be explained by their chaotropic influence on the "basic" free energies (Hill 1977; Läuger 1991) of the different conformational states of the protein. It has been shown in a number of studies that the conformation transition, $\mathrm{P}-\mathrm{E}_{1} / \mathrm{P}-\mathrm{E}_{2}$, is the rate limiting step of the electrogenic $\mathrm{Na}^{+}$transport, and that its rate constant controls the kinetics of the decay of the short circuit current (Holmgren et al. 2000; Sokolov et al. 1998a; Wuddel and Apell 1995). The observation that these anions modify the temperature dependence of $1 / \tau_{2}$, while the dependence of $1 / \tau_{1}$ is practically unaffected, supports this conclusion, since the rising phase with its time constant represents the (faster) preceding partial reactions, which includes ATP release from its caged form, ATP binding, and enzyme phosphorylation (Sokolov et al. 1998a). The effects of iodide were much stronger when it was added bilaterally, than when iodide was added after adsorption of the membrane fragments on the BLM, where it could affect only the cytoplasmic side of the protein. These experiments imply that the effect of iodide on the temperature dependence of $\tau_{2}$ is primarily due to its interaction with the extracellular parts of the protein.

The conformation transition, $\mathrm{P}-\mathrm{E}_{1} / \mathrm{P}-\mathrm{E}_{2}$, is known to open the access to the ion-binding sites from the extracellular aqueous phase through a so-called ion well. Therefore, it is possible that iodide (or bromide) affects the conformation transition by changing the free energy of the open-channel formation. The chaotropic effect of iodide and bromide can be related to changes of the energy necessary to bind water to the extracellular surface of the protein or to allow ions to enter the access channel. The fact that all the temperature dependences of the reciprocal time constants of $\tau_{2}$ intersect in the Arrhenius plot for the different iodide concentrations at the same temperature, the freezing point of water (Fig. 2a), implies that the effects of the anions on the time constant, $\tau_{2}$, vanished at this temperature. This observation supports the proposal of an effect on water binding to the protein surface. An alternative explanation of the observed effects, however, cannot be ruled out, such as specific binding of iodide (and bromide) to extracellular sites of the protein.

In the literature other possible explanations of the salt effects have been introduced. One proposal was an interaction of anions with the ATP-binding site-either by competitive binding or by shielding of electrostatic protein charges located in the vicinity of the binding center (Fedosova et al. 2003; Nørby and Esmann 1997). Neither mechanisms, however, can explain the iodide effects shown above: (1) significant effects occur already at low-iodide concentrations when the ionic strength of the solution remains practically unchanged, (2) the relaxation time $\tau_{2}$ is controlled by the conformation transition and not by enzyme phosphorylation, and (3) the ATP-binding site is situated on the cytoplasmic side of the membrane and is accessible for the chaotropic ions in the same way when added unilaterally or bilaterally, in contrast to the observation that iodide (or bromide) acted more efficiently when present at the extracellular side.

Alternative mechanisms stand to reason, however, in the case of the less chaotropic chloride ions. Significant chloride effects were observed only at higher concentrations when the ionic strength of the solution was enhanced considerably. Further support for an alternative mechanism is that chloride ions were more effective upon unilateral addition than upon bilateral addition (Fig. 5). Chloride ions did not measurably change the slope of the temperature dependence of $1 / \tau_{2}$, and therefore, the activation energy was not significantly modified (not shown). Finally, there is another important difference between the effects produced by chloride on the one hand and bromide or iodide on the other: Addition of the latter two anions to the buffer caused a decrease of the amount of electric charge transferred by the protein upon phosphorylation, whereas addition of 


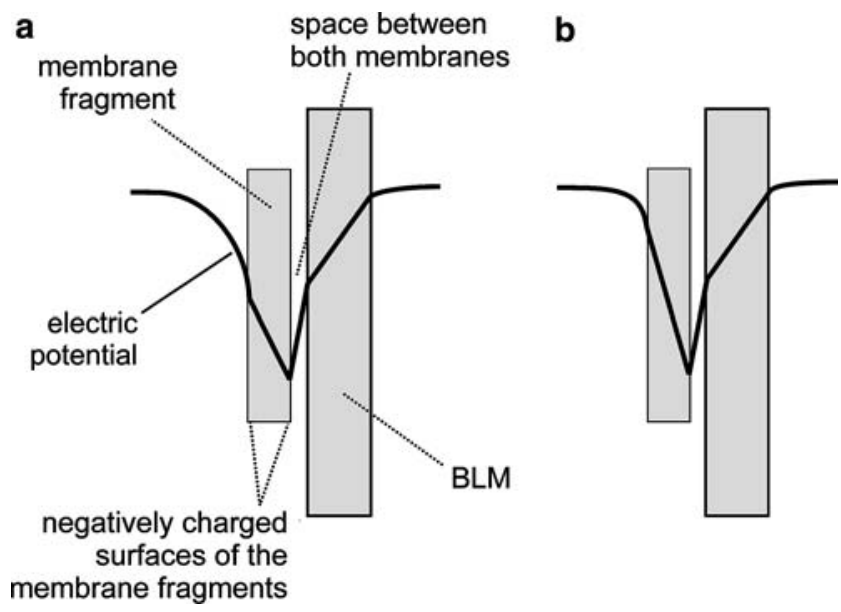

Fig. 7 Schematic representation of the electrostatic energy profile for cations across the BLM and an adsorbed membrane fragment in a solution of low-ionic strength (a) and after unilateral increase of ionic strength (b). The changes of the electrostatic potential have their origin in a shielding of the negative surface charges on the $\mathrm{Na}, \mathrm{K}-\mathrm{ATPase}$-containing membrane fragments by the increased ionic strength in the solution with a high-choline chloride concentration

chloride led even to a small increase (Fig. 6). The amount of the charge transferred depends on three factors: (1) the number of sodium ions transferred per protein molecule in response to the ATP-concentration jump, (2) the number of active protein molecules, and (3) how far the $\mathrm{Na}^{+}$transport is completed. There is no indication that the anions alter stoichiometry and functional activity of the Na,K-ATPase. In the absence of chaotropic anions the steady-state conformation after the relaxation process is almost completely state $\mathrm{P}-\mathrm{E}_{2}$ in the experimental conditions chosen. In the presence of the strongly chaotropic iodide, however, the $\mathrm{P}-\mathrm{E}_{1} / \mathrm{P}-\mathrm{E}_{2}$ conformational equilibrium is shifted in favor of the $\mathrm{E}_{1}$ conformation, this condition will prevent the (electrogenic) release of part of the $\mathrm{Na}$ ions, and in consequence, reduce the amount of charge transferred, as shown in Fig. 6. A mechanistic explanation for the observed shift towards the $\mathrm{E}_{1}$ state may be that the chaotropic anions produce a stronger decrease of the forward rate constant $\left(E_{1} \rightarrow E_{2}\right)$ than of the backward rate constant.

The increase of the number of $\mathrm{Na}$ ions transferred by the protein in the presence of chloride is most likely to be associated with the increased ionic strength of the electrolyte. In the case of an unilateral addition this leads to a decrease of the surface potential on the cytoplasmic side of the membrane due to shielding of the negative charges of lipid molecules on the cytoplasmic surface of the ATPase-containing membrane fragments. A change of the surface potential on the cytoplasmic side of the membrane can affect both the amount of ATP bound to the protein, and especially, the (apparent) transmembrane potential. This is illustrated by Fig. 7, a schematic representation of the electric-potential profile across the membrane fragments adsorbed to the BLM. A quantitative determination of the electric potentials occurring in such a system is hardly possible, and the profile depicted in Fig. 7 illustrates the two known facts only in principle. Firstly, both the data available from the literature and measurements of the electrophoretic mobility (unpublished results) indicate the presence of negatively charged lipids in the membrane fragments. In consequence, there is a potential change in the diffuse double layer at the interface between the membranes and the solution when the ionic strength is increased. Secondly, the membrane fragments and BLM are in close contact to each other. When the distance between the charged surface of fragments and BLM becomes comparable to the thickness of the diffuse double layer, a transmembrane-potential drop will occur to both membranes (Aityan et al. 1981). Figure 7a illustrates the potential profile at low-ionic strength, taking into account the surface charge of the fragment and the intramembrane potential drop in both membranes. An increase of ionic strength and the resulting decrease of the electrostatic surface potential on the electrolyte side of the membrane fragment modifies the potential distribution within it (Fig. 7b). Such an increase of the electric field inside the membrane is equivalent to an increase of the transmembrane potential, and causes an increase of the number of $\mathrm{Na}^{+}$ions transferred by the $\mathrm{Na}, \mathrm{K}-\mathrm{AT}$ Pase by a short-circuit current.

Thus, we have demonstrated that choline iodide and bromide not only decrease the rate of the rate-limiting step of the electrogenic ion transport, but also affect the activation energy of this step. This observation cannot be explained by the effect of the chaotropic anions on the phosphorylation step of the Na,K-ATPase, or by an significantly increased ionic strength that shields surface charges of the membrane (since, as we demonstrated in the case of choline chloride, the ionic strength has a different effect). The difference between the effects of unilateral and bilateral additions of iodide suggests that the primarily sensitive parts of the protein are located on the extracellular side. These results support the conclusion that the effects of iodide (and, to lesser degree, of bromide) are associated with an influence of the anions on the conformational transition. Since the $\mathrm{P}-\mathrm{E}_{1} / \mathrm{P}-\mathrm{E}_{2}$ conformation transition is known to open the extracellular ion-access channel, and alter the extracellular protein surface (Geibel et al. 2003), the iodide and bromide ions can be assumed to affect such modifications. A reasonable explanation for the observed effect is that chaotropic anions modify the intrusion of water molecules into the access channel and alter their coordination with the surface of the protein.

Acknowledgements The authors are grateful to $\mathrm{Yu}$. A. Chizmadzhev for critical discussion of the article, K. Pavlov and P. Kuzmin for the help in the preparing of the manuscript, and Milena Roudna for excellent technical assistance in protein preparation. The work was partly supported by the grants of the Support of Scientific Schools 1392.2003.4 and by INTAS 01-0224. 


\section{References}

Aityan SK, Belaya LM, Chizmadzhev YuA (1981) Interaction of membranes with constant surface charge. Biofizika 26:467-473

Apell H-J (2003) Toward an understanding of ion transport through the Na,K-ATPase. Ann NY Acad Sci 986:133-140

Borlinghaus R, Apell H-J, Läuger P (1987) Fast charge translocations associated with partial reactions of the Na,K-pump: I. Current and voltage transients after photochemical release of ATP. J Membr Biol 97:161-178

Deguchi N, Jørgensen PL, Maunsbach AB (1977) Ultrastructure of the sodium pump. Comparison of thin sectioning, negative staining, and freeze-fracture of purified, membrane-bound $\left(\mathrm{Na}^{+}, \mathrm{K}^{+}\right)$-ATPase. J Cell Biol 75:619-634

Fedosova NU, Champeil P, Esmann M (2003) Rapid filtration analysis of nucleotide binding to Na,K-ATPase. Biochemistry 42:3536-3543

Fendler K, Grell E, Haubs M, Bamberg E (1985) Pump currents generated by the purified $\mathrm{Na}^{+} \mathrm{K}^{+}$-ATPase from kidney on black lipid membranes. EMBO J 4:3079-3085

Fendler K, Jaruschewski S, Hobbs A, Albers W, Froehlich JP (1993) Pre-steady-state charge translocation in NaK-ATPase from eel electric organ. J Gen Physiol 102:631-666

Gadsby DC, Nakao M, Bahinski A, Nagel G, Suenson M (1992) Charge Movements via the cardiac Na,K-ATPase. Acta Physiol Scand 146:111-123

Ganea C, Babes A, Lüpfert C, Grell E, Fendler K, Clarke RJ (1999) Hofmeister effects of anions on the kinetics of partial reactions of the $\mathrm{Na}^{+}, \mathrm{K}^{+}$-ATPase. Biophys J 77:267-281

Geibel S, Kaplan JH, Bamberg E, Friedrich T (2003) Conformational dynamics of the $\mathrm{Na}^{+} / \mathrm{K}^{+}$-ATPase probed by voltage clamp fluorometry. Proc Natl Acad Sci USA 100:964-969

Hill TL (1977) Free energy transduction in biology. Academic Press, New York, pp 1-229
Holmgren M, Wagg J, Bezanilla F, Rakowski RF, de Weer P, Gadsby DC (2000) Three distinct and sequential steps in the release of sodium ions by the $\mathrm{Na}^{+} / \mathrm{K}^{+}$-ATPase. Nature 403:898-901

Jørgensen PL (1974) Isolation of $\left(\mathrm{Na}^{+}+\mathrm{K}^{+}\right)$-ATPase. Meth Enzymol 32:277-290

Klodos I, Post RL, Forbush B III (1994) Kinetic heterogeneity of phosphoenzyme of Na,K-ATPase modeled by unmixed lipid phases. Competence of the phosphointermediate. J Biol Chem 269:1734-1743

Läuger P (1991) Electrogenic ion pumps. Sinauer Assoc, Sunderland, MA, pp 1-313

Nørby JG, Esmann M (1997) The effect of ionic strength and specific anions on substrate binding and hydrolytic activities of Na,K-ATPase. J Gen Physiol 109:555-570

Pavlov KV, Sokolov VS (2000) Electrogenic ion transport by $\mathrm{Na}^{+}, \mathrm{K}^{+}$-ATPase. Membr Cell Biol 13:745-788

Post RL, Suzuki K (1991) A Hofmeister effect on the phosphoenzyme of Na,K-ATPase. Soc Gen Physiol Ser 46:201-209

Sokolov VS, Apell H-J, Corrie JE, Trentham DR (1998a) Fast transient currents in Na,K-ATPase induced by ATP concentration jumps from the P3-[1-(3',5'-dimethoxyphenyl)-2-phenyl2-oxo]ethyl ester of ATP. Biophys J 74:2285-2298

Sokolov VS, Ayuan AG, Apell H-J (2001) Assignment of charge movements to electrogenic reaction steps of the Na,K-ATPase by analysis of salt effects on the kinetics of charge movements. Eur Biophys J 515-527

Sokolov VS, Stukolov SM, Darmostuk AS, Apell H-J (1998b) Influence of sodium concentration on changes of membrane capacitance associated with the electrogenic ion transport by the Na,K-ATPase. Eur Biophys J 27:605-617

Wuddel I, Apell H-J (1995) Electrogenicity of the sodium transport pathway in the $\mathrm{Na}, \mathrm{K}-\mathrm{ATPase}$ probed by charge-pulse experiments. Biophys J 69:909-921 